\title{
Visualization of Spatial Distribution of Random Waypoint Mobility Models
}

\author{
Jiradett Kerdsri*, Tawiwat Veeraklaew \\ Defence Technology Institute, Nonthaburi, Thailand. \\ * Corresponding author. Tel.: +66909747183; email: jiradett.k@dti.or.th \\ Manuscript submitted November 5, 2015; accepted April 5, 2016. \\ doi: $10.17706 /$ jcp.12.4.309-316
}

\begin{abstract}
In multi-hop wireless network simulation, mobility model is one of the crucial factors affecting the performance of networks. This paper aims to analyze the spatial distribution of Random Waypoint mobility models with the progress of time. Additionally, we study the network performance of a random-based mobility model. The results from the evaluation show the uniform node distribution of Random Waypoint model in sparse network environment. In fact, we observe that nodes tend to be clustered in the middle with more number of nodes and progress of time. On the performance evaluation, the results show that Random Waypoint gain moderately higher delivery ratio than Random Walk and Random Direction models.
\end{abstract}

Key words: Random Waypoint, MANET, mobility model, wireless network.

\section{Introduction}

The most widely used mobility model for Mobile Ad- hoc Network (MANET) simulation is the Random Waypoint Model (RWP) [1] in which each node moves independently of each other in the obstacle-free environment. It is a simple stochastic mobility model to simulate the movement behavior of mobile nodes in two dimensional areas [2]. In fact, RWP is normally used in OppNets simulation such as in [3]-[6] where every node chooses a random location in the simulation area and moves towards it at a random speed uniformly chosen from $\left(V_{\min }, V_{\max }\right)$. The simplicity of analysis of this stochastic RWP has made it a benchmark for multi-hop mobile network simulation [7]. In the simulation, $V_{\min }$ is the minimum and $V_{\max }$ is the maximum speed of the nodes

In RWP model which is a generalization of Random Walk (RW), a new destination inside the network area is chosen randomly. Then the node moves towards that destination with a randomly selected speed as can be seen in the example topology and movement pattern in Fig. 1. Normally the Network Simulator implements this mobility model as follows:

- A node randomly chooses a destination and moves to-wards it with a velocity chosen uniformly and randomly from pre-defined ranges $\left[0, V_{\max }\right]$.

- The direction and velocity of a node are chosen independently of other nodes.

- Upon reaching the destination, the node stops for the pause time parameter, $T_{\text {pause, }}$ duration.

- After this duration, it again chooses a random destination and repeats the whole process again until the simulation ends.

Nevertheless, RWP suffer from the density wave phenomenon where the spatial node uniform distribution is transferred to a non-uniform distribution with the progress of time. Finally, it reaches the 
state where the node density is maximum at the center of the area while the node density is reduced to zero towards the border region as in Fig. 2 which shows the result of normalized distribution and contour line of certain occurrence values. Moreover, the distribution is symmetric in four axis direction of the center.

To the best of our knowledge, none of the RWP studies has been explored the nodal spatial behavior in order to visually capture the node distribution to analyze the affect on network performance. This paper aims to study the spatial node distribution of RWP while comparing its delivery ratio with other popular random based mobility models.

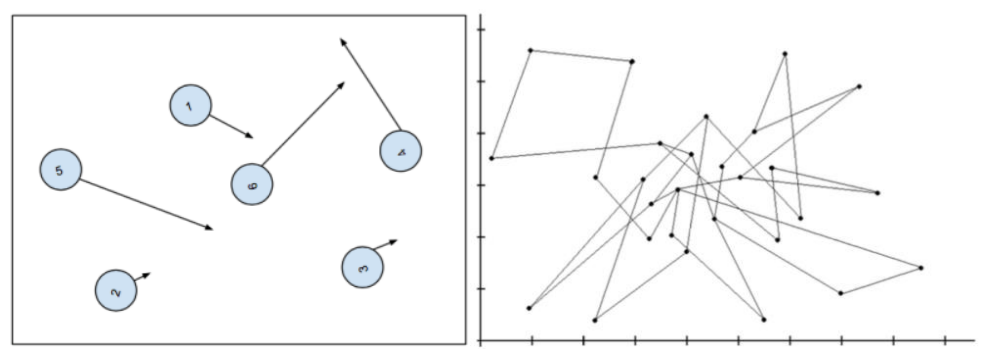

Fig. 1. Random Waypoint Model Example Topography and mobility movement pattern [8].

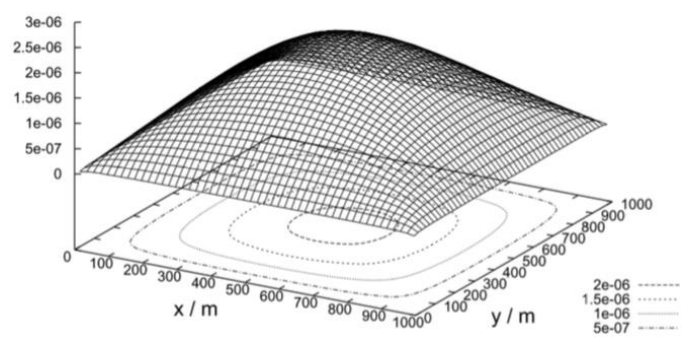

Fig. 2. Square simulation distribution [2].

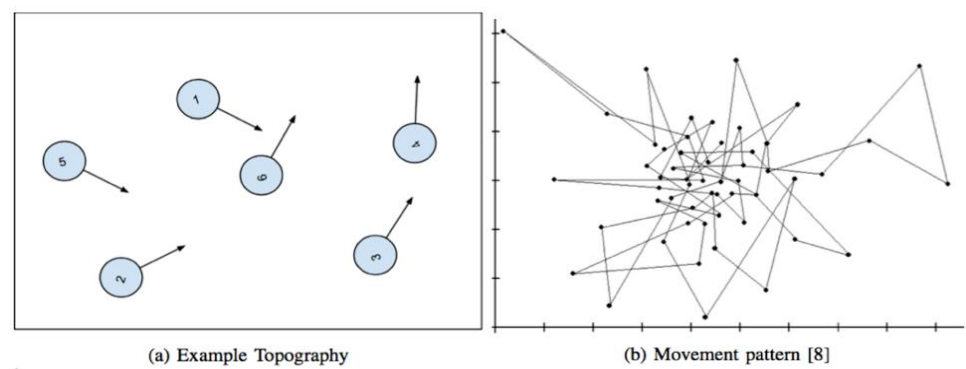

Fig. 3. Random walk model.

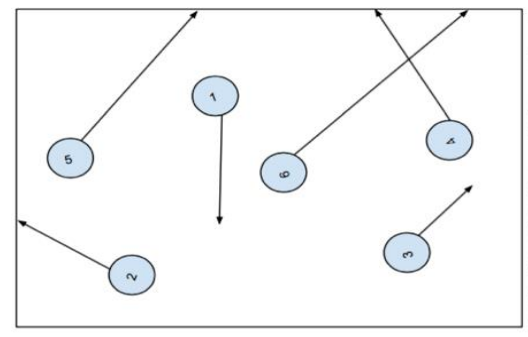

(a) Example Topography

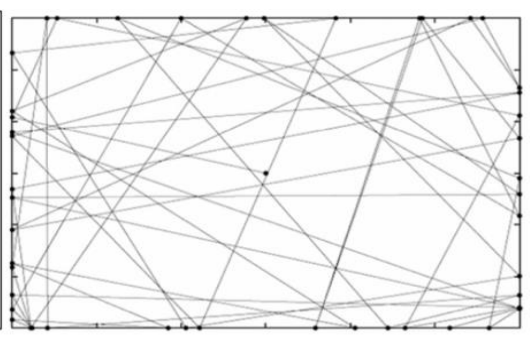

(b) Movement pattern $[8]$

Fig. 4. Random direction model.

\section{Literatures Review}

RWP was proposed in 1996 by D. Maltz and D. Johnson [9] and it has been one of the most popular synthetic mobility models for ad-hoc and sensor network simulations. Its example topology and movement 
pattern are shown in Fig. 1. Other important random based mobility model is the Random Walk (RW) which is a variant of the RWP. In RW, the mobile node moves from its current location to the new destination by selecting the random direction and speed. Each movement is occurring in a constant time interval, $t$, or a fix distant traveled, $d$, then each speed and direction are recalculated at the end of each move. The example topology and movement pattern of RW can be seen in Fig. 3. However, if the node reaches the simulation boundary, it will bounce of the border with the angle determined by its incoming direction [10].

However, the main problem of RWP is the density wave phenomenon where the mobile nodes are usually concentrate at the center of the simulation area with the progress of time. Then RWP may be harmful to some scenarios of opportunistic network simulations where the measurement requires higher degree of uniform spatial distribution [11]. As a result, the Random Direction (RD) is designed to avoid aforementioned problem. In RD, a node chooses a random direction to travel until it reaches the simulation area border. A node pauses for a redefined amount of time and then selects a new angular direction to start traveling again [12].

Nevertheless, some applications require the visualization of node distribution in order to capture the real behavior of the mobile nodes. As a result, this paper aims to snap the position of nodes on the different time using the simulator with the visual pane.

\section{Evaluations}

\subsection{Simulation Setup}

We conduct the extensive simulations using the Opportunistic Network Environment (ONE) simulator version 1.5.2 [13]. ONE is a powerful JAVA tool for generating different movement models, running simulation with various routing protocols, visualizing simulations in real time and generating results, and post processing the results. The simulation parameters are in Table 1.

Table 1. Simulation Variables

\begin{tabular}{ll}
\hline \hline Parameters & Configurations \\
\hline Operation Area & $1000 \mathrm{~m}^{2}$ \\
Router & Epidemic \\
Movement Model & Random Based \\
Simulation Time & $3600 \mathrm{~s}$. \\
\hline \hline
\end{tabular}

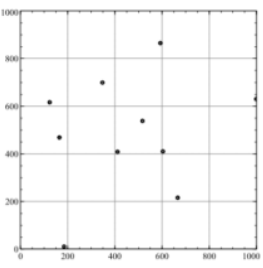

(a) $900 \mathrm{~s}$.

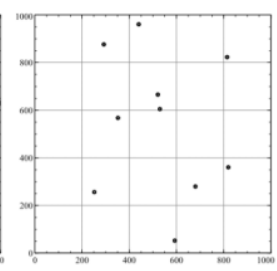

(b) $1800 \mathrm{~s}$

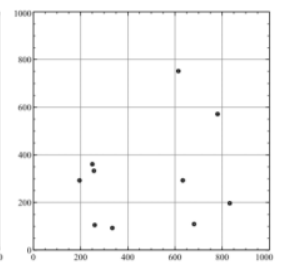

(c) $2700 \mathrm{~s}$

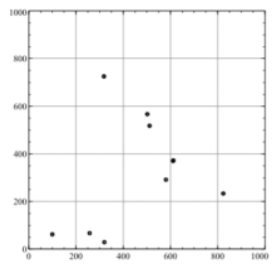

(d) $3600 \mathrm{~s}$.

Fig. 5. The distribution of 10 nodes at different time.

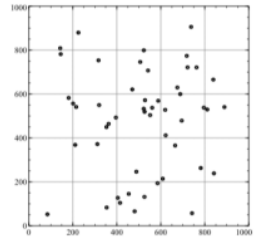

(a) $900 \mathrm{~s}$.

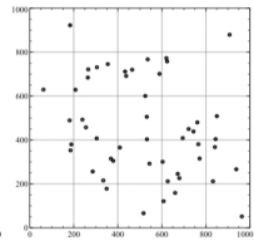

(b) $1800 \mathrm{~s}$

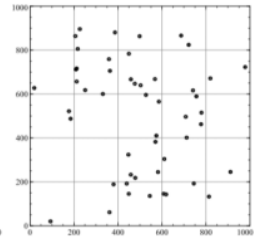

(c) $2700 \mathrm{~s}$.

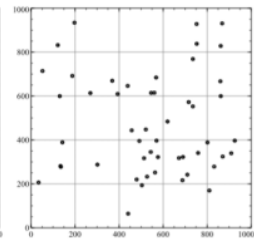

(d) $3600 \mathrm{~s}$.

Fig. 6. The distribution of 50 nodes at different time. 
Firstly, we aim to examine the node distribution on the different node density. We capture the snapshot of node distribution during each time interval during the simulation time varying by the number of nodes.

\subsection{Results}

Figs. 5 to 9 shows the 10, 50, 100, 200 and 300 nodes distribution in the operational area of $1000 \mathrm{~m}^{2}$ on the simulation time of $3600 \mathrm{~s}$. with $900 \mathrm{~s}$. interval. From visual observation, when the node density increase more than 35 nodes, most nodes tend to be more dense in the middle of the play-field comparing to the near border edges. On the other hand, nodes tend to be more uniformly distribution on the more sparse network environment (less then 35 nodes $/ \mathrm{km}^{2}$ in our experiment).

After we studied the relationship of node density on the node distribution, we aim to study the effect of simulation duration on the node distribution. Fig. 10 and Fig. 11 shows the 100 and 200 nodes distribution on the four different simulation time of $3600,36000,360000,3600000 \mathrm{~s}$. respectively. The graphs present that the nodes tend to be more slightly accumulate in the center with the progress of time. However, the differences in the node clustering overtime are not significant. Therefore, we can conclude that the nodes using RWP mobility models in the simulation tend to be accumulated in the center of the area in the dense networking environment while the duration of simulation has a minimal affect on the node clustering.

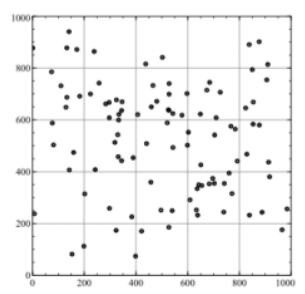

(a) $900 \mathrm{~s}$.

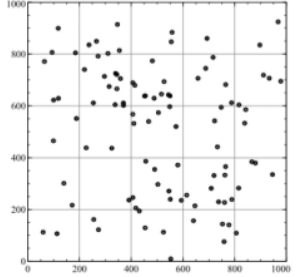

(b) $1800 \mathrm{~s}$.

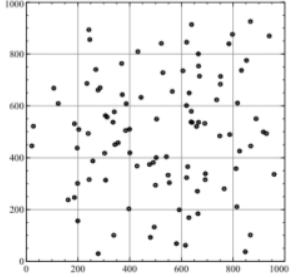

(c) $2700 \mathrm{~s}$.

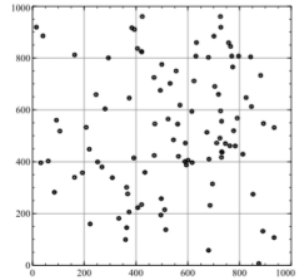

(d) $3600 \mathrm{~s}$.

Fig. 7. The distribution of 100 nodes at different time.

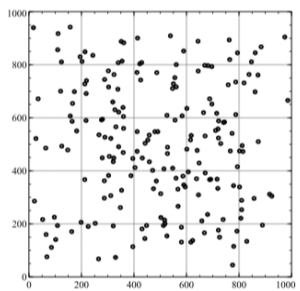

(a) $900 \mathrm{~s}$.

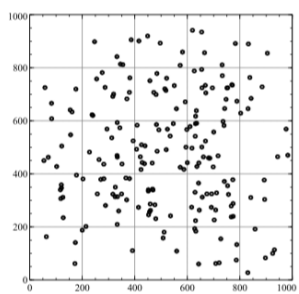

(b) $1800 \mathrm{~s}$.

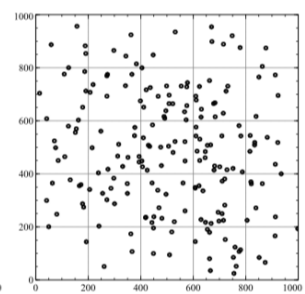

(c) $2700 \mathrm{~s}$.

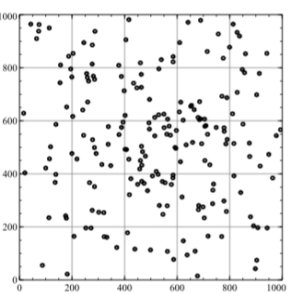

(d) $3600 \mathrm{~s}$.

Fig. 8. The distribution of 200 nodes at different time.

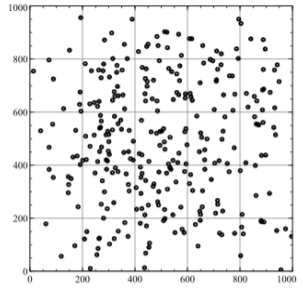

(a) $900 \mathrm{~s}$.

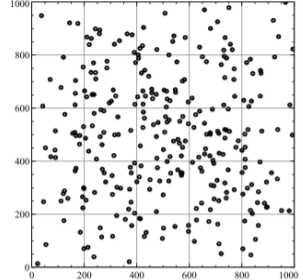

(b) $1800 \mathrm{~s}$.

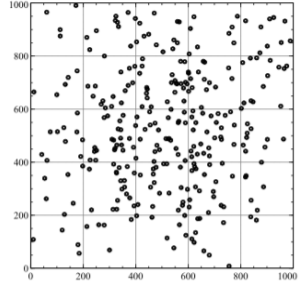

(c) $2700 \mathrm{~s}$

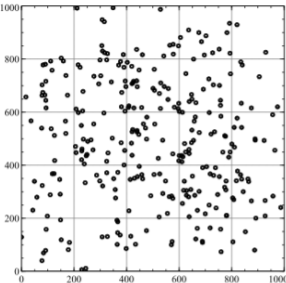

(d) $3600 \mathrm{~s}$.

Fig. 9. The distribution of 300 nodes at different time.

Consequently, we analyze the node distribution of the different mobility models: Random Waypoint, Random Walk (RW) and Random Direction (RD). Fig. 12-Fig. 14 show the distribution of 200 nodes at 4 different time interval. From visual observations, RWP comparing with RW and RD, tends to be more 
clustered in the center. On the other hand, RD tends to be uniformly distributed all over the area. In RW, the speed of a node is randomly selected and its direction is also randomly chosen. Then, each node goes in the selected random direction with the selected speed until the epoch lasts. Each epoch duration is again randomly selected as in Fig. 3 [14]. However, in RD model, a mobile node makes random mobility decisions with respect to current time or location, independent of other nodes. A node randomly picks a movement direction, and takes straight-line movement towards that direction for a given distance [15]. In this mobility model, the mobile node chooses any random direction to travel until the boundary of edge is found as in Fig. 4. In conclusion, the uniformly node distribution of RD is the result from the node movement to the border of the simulation area.

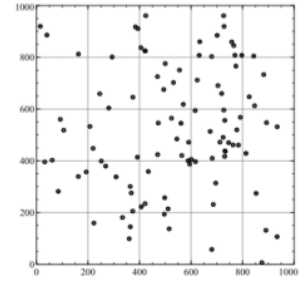

(a) $3600 \mathrm{~s}$.

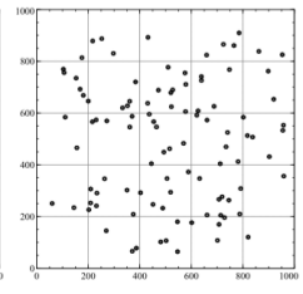

(b) $36000 \mathrm{~s}$.

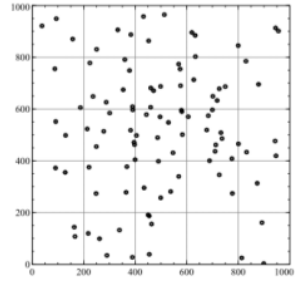

(c) $360000 \mathrm{~s}$.

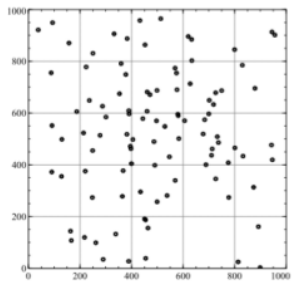

(d) $3600000 \mathrm{~s}$.

Fig. 10. The distribution of 100 nodes on different simulation duration.

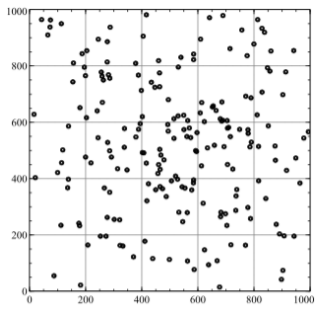

(a) $3600 \mathrm{~s}$.

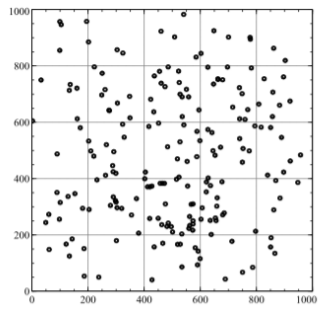

(b) $36000 \mathrm{~s}$.

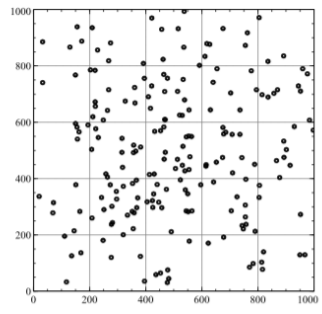

(c) $360000 \mathrm{~s}$.

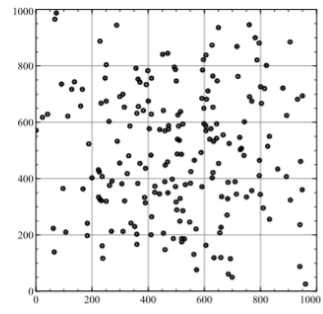

(d) $3600000 \mathrm{~s}$.

Fig. 11. The distribution of 200 nodes on different simulation duration.

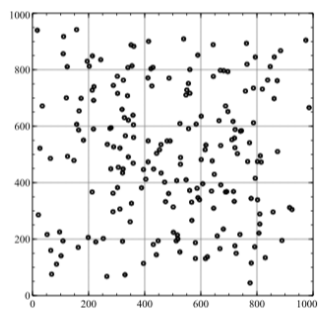

(a) $900 \mathrm{~s}$.

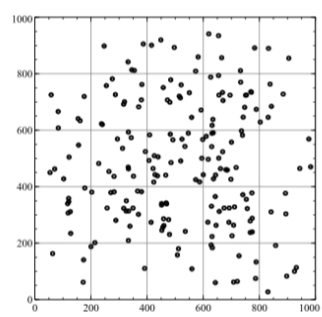

(b) $1800 \mathrm{~s}$.

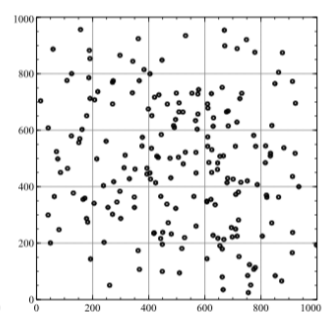

(c) $2700 \mathrm{~s}$.

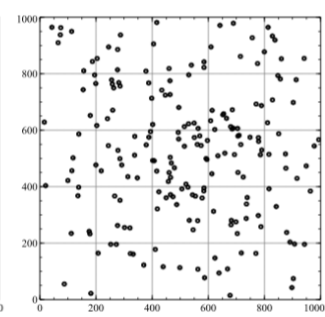

(d) $3600 \mathrm{~s}$.

Fig. 12. The distribution of 200 nodes on the Random Waypoint model at different time.

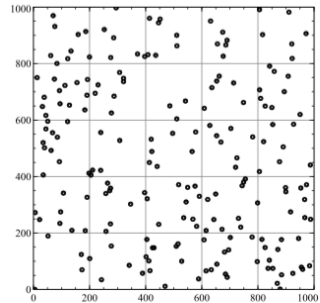

(a) $900 \mathrm{~s}$

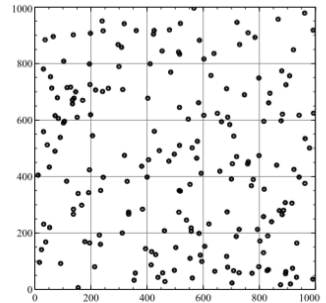

(b) $1800 \mathrm{~s}$.

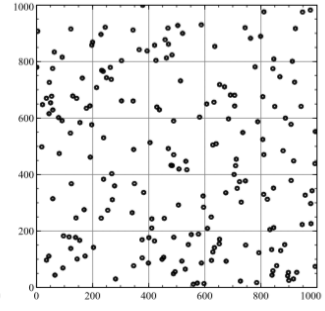

(c) $2700 \mathrm{~s}$.

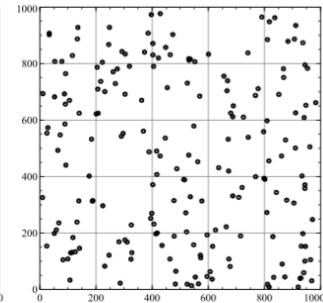

(d) $3600 \mathrm{~s}$.

Fig. 13. The distribution of 200 nodes on the Random Walk model at different time. 
Finally, we studied the performance of each mobility model. Fig. 15 shows the relationship of delivery ratio of Epidemic routing [16] on the node density. This graph presents the similar trend between RD, RWP and RW that the delivery ratio increases with number of nodes. Comparing from these three random mobility models, RWP gains slightly higher delivery ratio than RW and RD respectively, especially in the node density of $40-200$ nodes. When the nodes become more dense, the delivery ratios of all three random mobility models are getting similar.

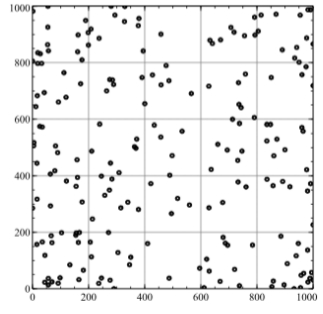

(a) $900 \mathrm{~s}$.

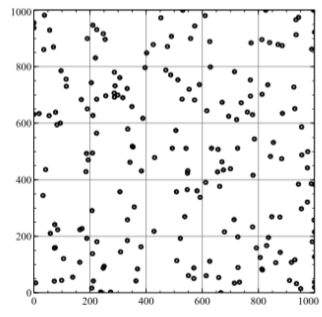

(b) $1800 \mathrm{~s}$.

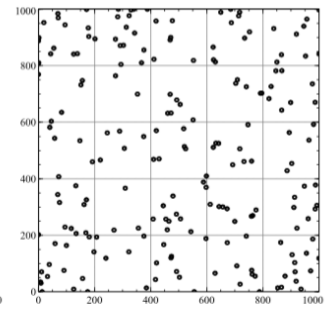

(c) $2700 \mathrm{~s}$.

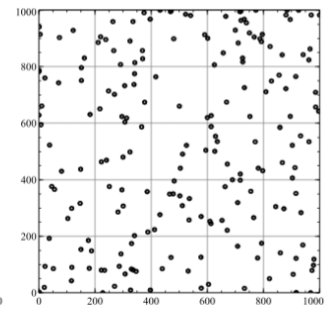

(d) $3600 \mathrm{~s}$.

Fig. 14. The distribution of 200 nodes on the Random Direction model at different time.

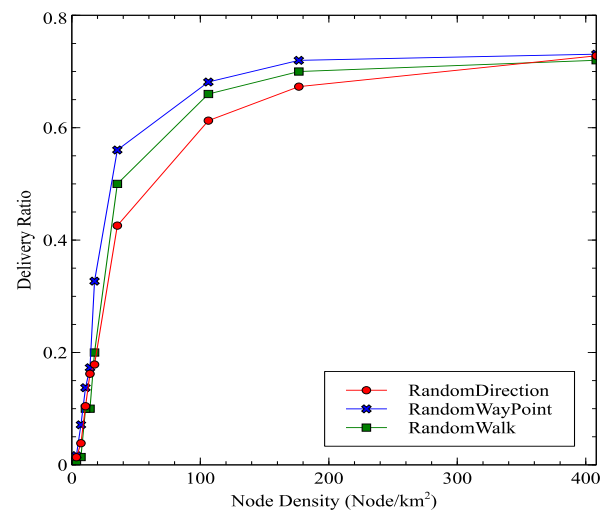

Fig. 15. Delivery Ratio on different mobility models.

\subsection{Discussion}

By visualize the spatial node distribution, we can clearly understand the behavior of the node movement. The result shows the tendency of node gathering at the center of playfield which can conform with density wave phenomenon that use the node frequency visit histogram. However, we suggest that the area of the operation might affect the node distribution since the border of playfield can cause the node to bounce back to the center of simulation area. Therefore, we can extend this work by study the impact of area size to the node distribution in the future work. In fact, the edge of playfield might never exist in the real scenario which can greatly alter the outcome of this study. We suspect that the node gathering behavior in the center might not occur in the simulation without the border. However, all the present simulation technology requires a specific limited space to simulate the movement environment. Thus, the non-boundary simulation cannot be performed with current simulation software technology.

The random direction movement model tends to be more uniformly distributed all over the area than the other movement models. This results in the lower in the delivery ratio than the others as well. We can observer that the centering cluster behavior greatly increase the delivery ratio which is impractical in some scenario applications. Therefore, we believe that the practical mobility model should be acquired from the real scenario gathering from the data from the actual environment.

\section{Conclusion}


The Random Waypoint is a popular model for simulation studies of wireless ad-hoc networks. This paper aims to investigate the spatial node distribution by visualization technique to capture the actual node movement behavior of this mobility model. From our extensive simulations, the mobile nodes tend to be more gathered in the center of the simulation area if the number of nodes and time increase. Therefore, the simulation with RWP model can be more realistically uniform distribution in sparse network environment. On the other hand, the nodes in Random Walk and Random Direction model are more uniformly distributed over the area while gain lower delivery ratio than RWP. For the future work, we can cooperate more complex visualization methods such as moving path or histogram of visited grids in order to gain more understanding of random based movement model behavior. In addition, the more advance simulation parameter should be performed to understand more behavior of node movement such as the the impact of area size, node speed, etc. Finally, the actual movement model gather from the real data should be also used in the simulation environment to extent the depth of anaysis.

\section{References}

[1] Rollo, M., \& Komenda, A. (2009). Mobility model for tactical networks. Lecture Notes in Computer Science (including subseries Lecture Notes in Artificial Intelligence and Lecture Notes in Bioinformatics), 5696 LNAI, 254-265.

[2] Bettstetter, C., \& Wagner, C. (2002). The spatial node distribution of the random waypoint mobility model. Proceedings of 1st German Workshop Mo- bile Ad-Hoc Network. WMAN (pp. 41-58).

[3] Shahbazi, S., Karunasekera, S., \& Harwood, A. (2012). Improving performance in delay/disruption tolerant networks through passive relay points. Wireless Networks.

[4] Yang, S., Yeo, C. K., \& Lee, B. S. (Jan. 2012). Toward reliable data delivery for highly dynamic mobile Ad Hoc networks. IEEE Transactions on Mobile Computing, 11(1), 111-124.

[5] Zhang, X. M., Wang, E. B., Xia, J. J., \& Sung, D. K. (Sept 2011). An estimated distance-based routing protocol for mobile ad-hoc networks. IEEE Transactions on, Vehicular Technology, 60(7), 3473-3484.

[6] Prodhan, A. T., Das, R., Kabir, H., \& Shoja, G. C. (2011). \{TTL\} based routing in opportunistic networks. Journal of Network and Computer Applications, 34(5), 1660-1670.

[7] Govindan, K., Chander, D., \& Jagyasi, B. (2010). Multihop Mobile Wireless Networks, River Publishers series in communications. River Publishers.

[8] Almeida e Silva, A. M. de. (2008). Simulation of Telecommunication Networks for Public Transportation Systems, Universidade do Porto.

[9] Johnson, D., \& Maltz, D. (1996). Dynamic source routing in ad hoc wireless networks. The Kluwer International Series in Engineering and Computer Science, 353, 153-181, Springer US.

[10] Martyna, J. (2012). Communications in Computer and Information Science, 291, 324-333, Springer Berlin Heidelberg.

[11] Bettstetter, C., Resta, G., \& Santi, P. (July 2003). The node distribution of the random waypoint mobility model for wireless ad-hoc networks. IEEE Transactions on Mobile Computing, 2(3), 257-269.

[12] Martyna, J. (2012). Simulation study of the mobility models for the wireless mobile Ad Hoc and sensor networks, Springer, 324-333.

[13] Keranen, A., Ott, J., \& Kärkkäinen, T. (2009). The ONE simulator for DTN protocol evaluation, Proceedings of the Second International ICST Conference on Simulation Tools and Techniques.

[14] Bulut, E. (2011). Opportunistic Routing Algorithms. Ph.D. dissertation, Rensselaer Polytechnic Institute.

[15] Thakur, G., Kumar, S. U., Helmy, A., \& Hsu, W. J. (July 2011). On the efficacy of mobility modeling for DTN evaluation: Analysis of encounter statistics and spatiotemporal preferences. Proceedings of the 2011 7th International Wireless Communications and Mobile Computing Conference (pp. 510-515). 
[16] Vahdatand, A., \& Becker, D. (2000). Epidemic routing for partially-connected Ad Hoc networks. Technical Report CS-200006, Duke University, Tech. Rep.

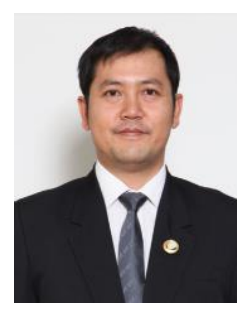

Jiradett Kerdsri received the B.E. (civil) degree from Royal Thai Air Forces Academy, the M.S. and M.E. degree from Naval Postgraduate School, USA and University of Melbourne, Australia both in computers. He received the Ph.D. degree in information computer technology from Sirindhorn International Institute of Technology (SIIT). He is a director of data communication Laboratory at Defence Tehcnology Institute under the Ministry of Defense. His research interests include opportunistic networks, wireless networks and computer communications.

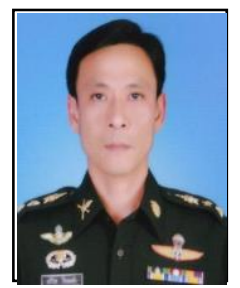

Tawiwat Veeraklaew received the Ph.D. degree in mechanical engineering from University of Delaware, Newark, DE, USA in 2000. He is a Deputy Director-General at Defence Technology Institute (Public Organization), Nontburi, Thailand. He has published more than 30 both in conference and journal articles. His current research interests are in the area of controlled mechanical systems, dynamic optimization and special software hardware design. 